\title{
Spectropolarimetry of Primitive Phototrophs as Global Surface Biosignatures
}

\author{
William B. Sparks, ${ }^{1,2}$ Mary Niki Parenteau, ${ }^{3,4}$ Robert E. Blankenship,,${ }^{3,5,6}$ Thomas A. Germer, ${ }^{7}$ \\ Christian Herman Lucas Patty, ${ }^{8,9}$ Kimberly M. Bott, ${ }^{3,10}$ Charles M. Telesco, ${ }^{11}$ and Victoria S. Meadows ${ }^{3,12}$
}

\begin{abstract}
Photosynthesis is an ancient metabolic process that began on early Earth and offers plentiful energy to organisms that can utilize it such that that they achieve global significance. The potential exists for similar processes to operate on habitable exoplanets and result in observable biosignatures. Before the advent of oxygenic photosynthesis, the most primitive phototrophs, anoxygenic phototrophs, dominated surface environments on the planet. Here, we characterize surface polarization biosignatures associated with a diverse sample of anoxygenic phototrophs and cyanobacteria, examining both pure cultures and microbial communities from the natural environment. Polarimetry is a tool that can be used to measure the chiral signature of biomolecules. Chirality is considered a universal, agnostic biosignature that is independent of a planet's biochemistry, receiving considerable interest as a target biosignature for life-detection missions. In contrast to preliminary indications from earlier work, we show that there is a diversity of distinctive circular polarization signatures, including the magnitude of the polarization, associated with the variety of chiral photosynthetic pigments and pigment complexes of anoxygenic and oxygenic phototrophs. We also show that the apparent death and release of pigments from one of the phototrophs is accompanied by an elevation of the reflectance polarization signal by an order of magnitude, which may be significant for remotely detectable environmental signatures. This work and others suggest that circular polarization signals up to $\sim 1 \%$ may occur, significantly stronger than previously anticipated circular polarization levels. We conclude that global surface polarization biosignatures may arise from anoxygenic and oxygenic phototrophs, which have dominated nearly $80 \%$ of the history of our rocky, inhabited planet. Key Words: Polarimetry-Biosignatures-Exoplanets. Astrobiology 21, 219-234.
\end{abstract}

\section{Introduction}

T HE DISCOVERY OF thousands of exoplanets and the consequent implication that there are large numbers of habitable environments throughout the Galaxy raises the fundamental question, "Are these planets in fact inhabited?"
Efforts to resolve this culturally profound and scientifically vital issue are driving an explosive growth in exoplanet research and technology development, aimed at directly imaging and characterizing Earth-like exoplanets. Ancillary research into the origins of planetary systems and comparative planetology to establish an accurate context for

\footnotetext{
${ }^{1}$ SETI Institute, Mountain View, California, USA

${ }^{2}$ Space Telescope Science Institute, Baltimore, Maryland, USA.

${ }^{3}$ Virtual Planetary Laboratory, University of Washington, Seattle, Washington, USA.

${ }^{4}$ NASA Ames Research Center, Moffett Field, California, USA.

Departments of ${ }^{5}$ Biology and ${ }^{6}$ Chemistry, Washington University in St. Louis, St. Louis, Missouri, USA.

${ }^{7}$ National Institute of Standards and Technology, Gaithersburg, Maryland, USA.

${ }^{8}$ Institute of Plant Biology, Hungarian Academy of Sciences, Szeged, Hungary.

${ }^{9}$ Space Research and Planetary Sciences, University of Bern, Bern, Switzerland.

${ }^{10}$ Department of Earth and Planetary Sciences, University of California, Riverside, Riverside, California, USA.

${ }^{11}$ Department of Astronomy, University of Florida, Gainesville, Florida, USA.

${ }^{12}$ Department of Astronomy, University of Washington, Seattle, Washington, USA.

(C) William B. Sparks et al., 2020; Published by Mary Ann Liebert, Inc. This Open Access article is distributed under the terms of the Creative Commons Attribution Noncommercial License (http://creativecommons.org/licenses/by-nc/4.0/) which permits any noncommercial use, distribution, and reproduction in any medium, provided the original author(s) and the source are credited.
} 
understanding our place in the habitable and uninhabitable Universe is likely to represent a major endeavor in the coming decades.

To determine whether an exoplanet hosts life, we expect to rely on remotely detectable biosignatures, observational signals that are produced by living organisms that can be seen from astronomical distances with suitable telescopes. Ideally, these signatures would be uniquely produced by a biological agent, but in practice, we need to account for the probability of abiotic production (Des Marais et al., 2008; Schwieterman et al., 2018). Although much emphasis has been placed on spectroscopic characterization of planetary atmospheres, derivation of atmospheric composition and its interpretation in terms of a biosphere requires observation over a wide wavelength range, extensive modeling, and the elimination of numerous potential "false-positive" signals (e.g., Meadows et al., 2018).

A more direct approach is afforded by the concept of "surface biosignatures," where an observable characteristic of the directly observed biological material serves as a biosignature, with the likelihood of reduced model dependence (Schwieterman et al., 2018). Photosynthetic utilization of the host star's radiant energy is, by necessity, a surface phenomenon. Further, the interaction of biological material with the incident light through the intermediary of its pigments is a strong interaction rendered polarizationsensitive by chiral structures within the pigments and pigment complexes (Sparks et al., 2009a, 2009b; Patty et al., 2018; Schwieterman et al., 2018). Hence, photosynthetic communities, which have the potential to achieve global environmental dominance, also have the potential to exhibit surface biosignatures through their spectral and circular polarization characteristics. That is, circular polarization spectroscopy is potentially a powerful surface biosignature.

Further, the uniquely biological phenomenon of homochirality is considered a universal biosignature that is independent of a planet's biochemistry (Wald, 1957; Sparks et al., 2009a; MacDermott, 2012; Patty et al., 2018; Glavin et al., 2020). A molecule is considered chiral if it is not superimposable on its mirror image. Abiotic chiral molecules exist, but the abiotic synthesis of equal proportions of each "handedness" of the molecules leads to what is termed a racemic mixture. In contrast, biological molecules and polymers are built from a single handedness or enantiomer (e.g., left-handed amino acids and right-handed sugars). Members of each of the biological macromolecular groupsproteins, carbohydrates, nucleic acids, and lipids_-are chiral and, in practice, complex biological molecules typically contain multiple chiral centers. Here, we use the term "chirality" to cover the complexity of homochiral phenomena exhibited in biological systems, from the individual molecular level to supramolecular assemblies. It is an open question in the origins of life research as to how enantiomeric excess and homochirality first arose, but it is considered a universal or agnostic trait of all biochemical life.

The individual molecules, as well as polymers and macromolecules composed of unique enantiomers of their constituent monomers, are typically optically active and rotate polarized light in either a clockwise or counterclockwise direction, dependent on wavelength (optical rotatory dispersion), or preferentially absorb left or right circularly polarized light (circular dichroism, CD). Polarization sig- natures offer a well-known probe of biomolecular structure (Kelly and Price, 2000), and they have been shown to be present in pure microbial cultures (Sparks et al., 2009a, $2009 \mathrm{~b}$ ), and in more complex structures such as vegetation and algae (Patty et al., 2017, 2019). With the capacity for circular polarization spectroscopy to detect chirality, without requiring molecular specificity of many biologically motivated life-detection tests, circular polarization spectroscopy, therefore, has the potential to be a generic, agnostic (universal) biosignature.

It appears likely that circular polarization biosignatures are free from abiotic mimics (Sparks et al., 2009a, 2009b), and they can be distinguished from circular polarization originating from multiple scattering effects by clouds or other aerial particles, which create a weak signal that is spectrally very flat (Rossi and Stam, 2018). Distinctive polarization features due to biological pigments and pigment complexes potentially allow us to probe exoplanet environments across, essentially, the entirety of their living history with a plausibly pure biosignature, free from many of the model dependencies of atmospheric and surface spectroscopy.

The circular polarization spectrum can provide a remote sensing and in situ capability to infer the presence of chiral biological material. For life-detection missions, finding an enantiomeric excess $\geq 20 \%$ of one handedness suggests the presence of a biological source (Neveu et al., 2018; Glavin et al., 2020). Acquisition of circular polarization spectra is likely to include incidentally both precision spectroscopy and linear polarimetry. High signal-to-noise telescopic spectroscopy can reveal classical atmospheric biogenic gases, whereas linear polarimetry will characterize clouds, rainbows, liquids, solids, and aerosols in the scattered light of the planet. Taken altogether, such observations would provide an effective diagnostic suite to answer the difficult question of whether a particular remote target hosts life, once large, capable, probably space-based, telescopes become available. Within the Solar System, both remote sensing and in situ measurements are feasible and targets such as the plumes and surfaces of icy moons, impact craters on Mars, the organic haze of Titan's atmosphere, and the clouds of Venus offer example destinations.

Indeed, chirality detectors have already flown within the Solar System, although a victim of unrelated technical malfunctions (McKay et al., 1998; Goesmann et al., 2015), whereas new ones are being prepared for upcoming missions (Goesmann et al., 2017). The search for homochirality has also been explicitly proposed for extrasolar life detection (MacDermott, 1998).

In this work, we characterize the spectropolarimetric surface biosignatures associated with a variety of anoxygenic phototrophs and oxygenic phototrophs (cyanobacteria). Anoxygenic photosynthetic bacteria represent evolutionary precursors to the oxygenic photosynthetic cyanobacteria, deriving and storing energy from sunlight, synthesizing ATP by cyclic photophosphorylation without the production of oxygen. Anoxygenic photosynthetic microbes have been present for $\sim 80 \%$ of the history of life on Earth and, hence, provide an attractive target to probe epochs before the rise of biogenic atmospheric gases, as well as a surface biosignature subsequently. Blankenship (2014) reviews the evolution and history of photosynthesis. The major pigments of 
Table 1. Pure Cultures of the Five Major Groups of Anoxygenic Phototrophs Containing Bacteriochlorophyll Pigments Analyzed in This Study

\begin{tabular}{|c|c|c|c|}
\hline Pure culture & $\begin{array}{l}\text { Photosynthetic } \\
\text { group }\end{array}$ & Pigments & Environmental source \\
\hline Thermochromatium tepidum, type strain (DSM 3771) & Purple sulfur & Bchl $a$ & Continental hot spring \\
\hline Rhodobacter capsulatus strain SB 1003 (ATCC BAA-309) & Purple non-sulfur & Bchl $a$ & Soil \\
\hline Prosthecochloris aestuarii $2 \mathrm{~K}$ & Green sulfur & $\begin{array}{l}\text { Bchl } c, a ; \\
\quad \text { Chl } a\end{array}$ & Marine intertidal lagoon \\
\hline Chlorobium tepidum TLS, Type strain (ATCC 49652) & & $\mathrm{Bchl} c, a$ & Continental hot spring \\
\hline Chloroflexus aurantiacus J-10-fl & FAPs & $\begin{array}{l}\mathrm{Bchl} c, a ; \\
\text { Bchl } a\end{array}$ & Continental hot spring \\
\hline Roseiflexus castenholzii HLO8, type strain (DSM 13941) & FAPs & Bchl $a$ & Continental hot spring \\
\hline $\begin{array}{l}\text { Heliobacterium modesticaldum Ice1, type strain } \\
\text { (ATCC 51547) }\end{array}$ & Heliobacteria & Bchl $g$ & Continental hot spring \\
\hline
\end{tabular}

Bchl, bacteriochlorophyll; Chl, chlorophyll; FAP, filamentous anoxygenic phototroph.

photosynthesis, bacteriochlorophylls (Bchls) and chlorophylls (Chls), are optically active molecules with chiral centers, illustrated, for example, in the work of Senge et al. (2014), their figure 6. These pigments absorb the visible and near-infrared (NIR) from $\sim 370$ to $1040 \mathrm{~nm}$, and they are well suited to absorbing light from a variety of stellar types (e.g., $\mathrm{G}$ stars and $\mathrm{M}$ stars). In addition to the intrinsic chirality of the pigments, larger conformational pigment and pigment-protein complexes can be chiral. An interesting phenomenon arises from large and dense chiral supramolecular assemblies, such as can be found in vegetation and algae. Such chiral aggregates can cause very intense polarization signals with non-conservative anomalously shaped bands that can extend beyond the molecular absorption bands (Keller and Bustamante, 1986; Garab and van Amerongen, 2009). The characterization of samples of such organisms offers encouragement that surface biosignature exploration of distant planets may be possible for a large fraction of their history and that for both extrasolar planets and within the Solar System, free from terrestrial assumptions and specificity, we may make significant progress in agnostic life detection by using circular polarization spectroscopy.
Section 2 presents our methodology, Section 3 provides the empirical results, Section 4 discusses the measurements and their relevance to life detection, and Section 5 presents the conclusion.

\section{Methods}

\subsection{Samples}

We measured the in vivo absorbance spectra and linear and circular polarization spectra of pure cultures of photosynthetic microbes and environmental photosynthetic microbial mats. Pure cultures of representatives of the five major anoxygenic photosynthetic groups were analyzed in this study (Table 1 and Fig. 1). The recently described sixth photosynthetic group, including Chloracidobacterium thermophilum, was not analyzed (Bryant et al., 2007). Cultures were obtained from the Blankenship lab, which maintains them for various pigment and reaction center studies. Many of the anoxygenic phototrophs are highly characterized type strains. Chloroflexus aurantiacus J-10-fl was obtained from B. Pierson (Pierson \& Castenholz 1974), and it is maintained at NASA Ames Research Center by Parenteau.
FIG. 1. Pure cultures of anoxygenic phototrophs analyzed in this study.

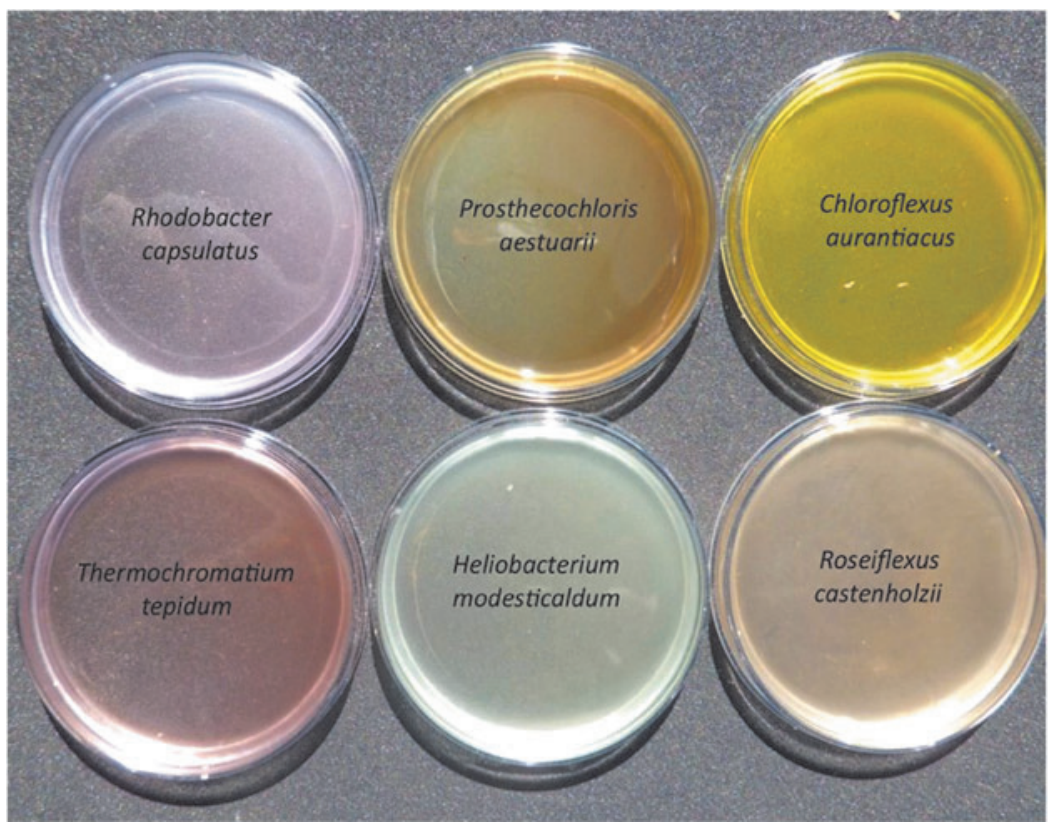


Table 2. Composition of Microbial Mats Analyzed in This Study

\begin{tabular}{|c|c|c|c|}
\hline Microbial mat type & Major phototrophic representatives & Pigments & Environmental source \\
\hline Mastigocladus streamers & $\begin{array}{l}\text { Cyanobacteria (Mastigocladus sp.), } \\
\text { anoxygenic phototrophs } \\
\text { (Chloroflexus sp.) }\end{array}$ & $\begin{array}{l}\text { Carotenoids, Chl } a \text {; } \\
\quad \text { Bchl } c, a\end{array}$ & Continental hot spring \\
\hline Phormidium mat & $\begin{array}{l}\text { Cyanobacteria (Phormidium or } \\
\text { Leptolyngbya sp.), anoxygenic } \\
\text { phototrophs (Chloroflexus sp., } \\
\text { Roseiflexus sp.) }\end{array}$ & $\begin{array}{l}\text { Carotenoids, Chl } a \text {; } \\
\quad \text { Bchl } c, a\end{array}$ & Continental hot spring \\
\hline Oscillatoria princeps mat & Cyanobacteria $(O$. princeps $)$ & $\begin{array}{l}\text { Carotenoids, phycocyanin, } \\
\text { Chl } a \text {; Bchl } c, a\end{array}$ & Continental hot spring \\
\hline $\begin{array}{l}\text { Synechococcus-Chloroflexi } \\
\text { mat }\end{array}$ & $\begin{array}{l}\text { Cyanobacteria (Synechococcus } \\
\text { sp. Cyanothece sp.), anoxygenic } \\
\text { phototrophs (Chloroflexus sp., } \\
\text { Roseiflexus sp.) }\end{array}$ & $\begin{array}{l}\text { Carotenoids, Chl } a \\
\quad \text { Bchl } c, a\end{array}$ & Continental hot spring \\
\hline Chloroflexi red underlayer & $\begin{array}{l}\text { Anoxygenic phototrophs } \\
\quad \text { (Chloroflexus sp., Roseiflexus sp.) }\end{array}$ & $\begin{array}{l}\text { Carotenoids, Chl } a \text {; } \\
\text { Bchl } c, a\end{array}$ & Continental hot spring \\
\hline $\begin{array}{l}\text { Chocolate Pots } \\
\text { Synechococcus- } \\
\text { Chloroflexi mat }\end{array}$ & $\begin{array}{l}\text { Cyanobacteria (Synechococcus sp., } \\
\text { Cyanothece sp.), anoxygenic } \\
\text { phototrophs (Chloroflexus sp., } \\
\text { Roseiflexus sp.) }\end{array}$ & $\begin{array}{l}\text { Carotenoids, Chl } a \text {; } \\
\quad \text { Bchl } c, a\end{array}$ & Continental hot spring \\
\hline
\end{tabular}

On the basis of its differing absorption and reflection polarization spectra, Section 3.1.3 given later, Chlorobaculum tepidum was selected for further study and an increased sample size of the pure culture was harvested at the exponential phase from the Blankenship laboratory and shipped to NIST following standard protocols in January 2016 (the "Jan16" sample). When we attempted to measure the Jan16 sample, we experienced a hardware problem with the polarimeter, requiring a period of several months for recovery of the instrument. After replacement of the internal monochromator, the instrument was brought back into service and was found to function to the same level of accuracy as before the failure. Measurements of the Jan16 sample eventually commenced during May 2016. For the intervening 4 months, the samples were stored in a freezer at NIST. When thawed out, these samples exhibited clear indication of cell lysis leading to the extracellular release of pigments.

Samples of photosynthetic microbial mats were obtained from Yellowstone National Park under Parenteau's permit number 1549 . These laminated mats contain cyanobacteria in the surface layer, followed by underlayers of anoxygenic phototrophs and chemotrophs (Table 2 and Fig. 2). The mats contained genera represented by the pure cultures (e.g., Chloroflexus and Roseiflexus), and the pure cultures were used to help deconvolve the polarization signals of the complex natural mats. The mats were harvested in the field, immediately placed on dry ice, then stored at $-80^{\circ} \mathrm{C}$ in the laboratory, and finally shipped on dry ice to NIST for the circular polarization measurements. After freezing, storage, and transportation to NIST - they were not measured in situthe mats were measured by using the same configuration as that of the reflection measurements for the pure cultures.

\subsection{Pigment analyses}

The in vivo absorption spectra of the pure cultures and photosynthetic microbial mats were obtained by sonicating the cells in cold Tris-sodium-magnesium (TSM) buffer. The cohesive mats were first dispersed with a glass tissue homogenizer before sonication to ensure total disruption of the cells to release the pigment-protein complexes. After sonication, the cellular debris was removed by centrifugation. The absorption of the pigment-protein complexes was measured from 350 to $1100 \mathrm{~nm}$ on a Shimadzu UV-1601 scanning spectrophotometer (Pierson and Parenteau, 2000). The purpose in performing the in vivo pigment analyses independent of the polarization measurements was to identify the major absorption peaks with traditional microbiological methods to help deconvolve the spectral features in the circular polarization spectra.

\subsection{Measurement of the polarization spectra}

2.3.1. Polarimetric instrumentation. The polarimeter and measurement methods are described in detail in the work of Sparks et al. (2009a). The instrument is a Hinds Instruments, Series II/FS42-47 dual photoelastic modulator (PEM) full Stokes polarimeter optimized for measurement of circular polarization in the presence of significant linear polarization. The polarimeter has a spectral resolution of $\sim 10 \mathrm{~nm}$ full width half maximum and covers the spectral range 400$800 \mathrm{~nm}$ by scanning in $5 \mathrm{~nm}$ steps. The polarization is described by the Stokes vector $S \equiv(I, Q, U, V) \equiv I(1, q, u, v)$, where $I$ is the total intensity, $Q$ and $U$ are the unnormalized linear Stokes parameters, $V$ is the unnormalized circular polarization Stokes parameter, and $q, u$, and $v$ are the normalized linear and circular Stokes parameters. The degree of linear polarization is $p_{l}=\sqrt{q^{2}+u^{2}}$, the linear polarization position angle is $\zeta=\frac{1}{2} \tan ^{-1}(u / q)$, and the degree of circular polarization is $v$. (The degree of polarization is 1 or $100 \%$ when perfectly polarized.) The target performance of measuring a degree of circular polarization $v=10^{-4}$ in the presence of linear polarization degree $p_{l}=0.03$ is achieved. Since the linear and circular polarization data are carried on the $2 f$ and $1 f$ modulation frequencies of the PEM resonant frequency $f$, respectively, there is no cross-talk between the linear and circular polarization measurements.

2.3.2. Sample measurement methods. Each sample of the pure cultures and photosynthetic microbial mats was 

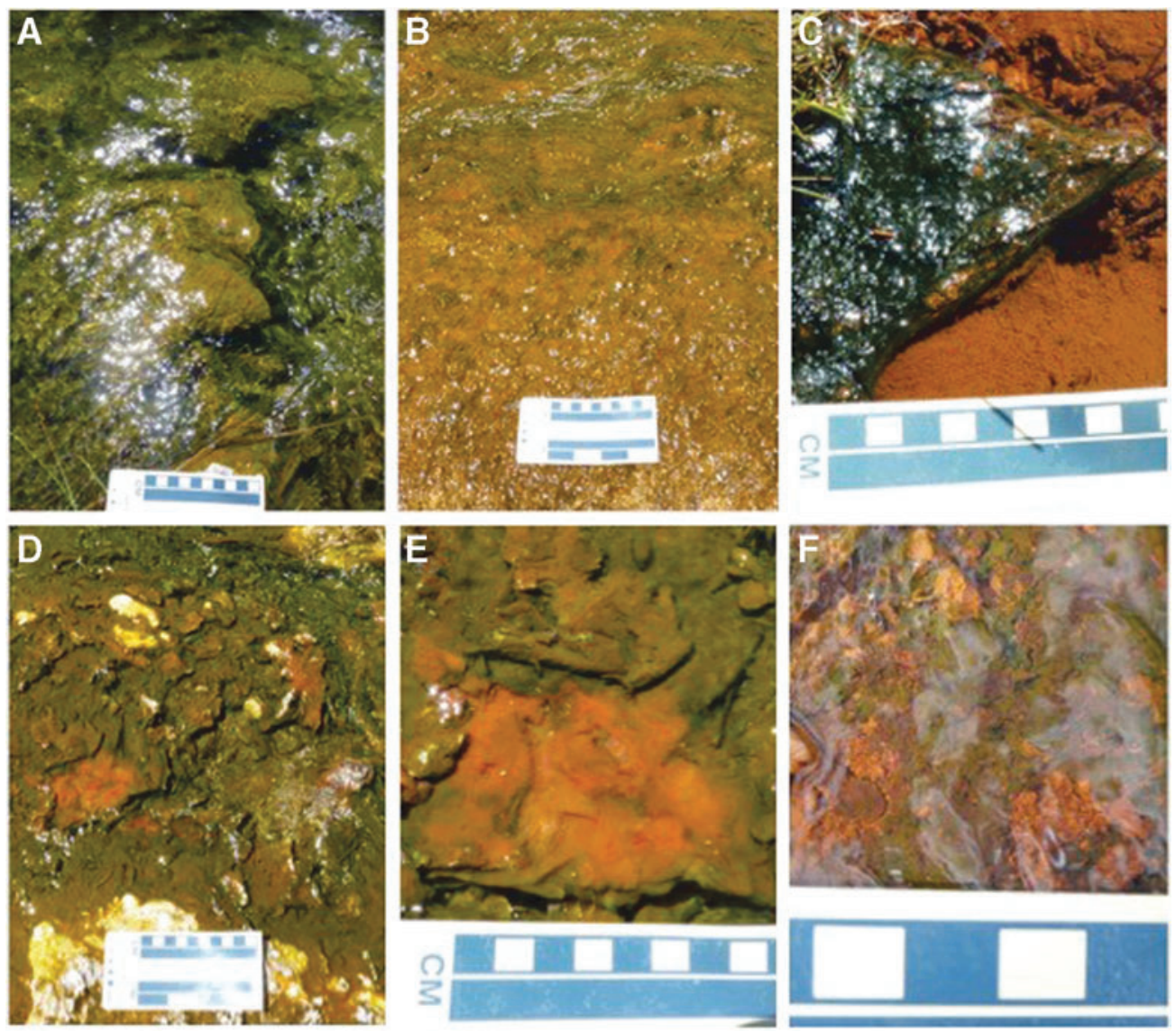

FIG. 2. Field photos of photosynthetic mats in the studied locations. (A) White Creek Mastigocladus streamers, (B) White Creek Phormidium mat, (C) Chocolate Pots Oscillatoria princeps mat on an iron mineral substrate, (D) White Creek Synechococcus-Chloroflexi mat, (E) White Creek Chloroflexi red underlayer, (F) Chocolate Pots SynechococcusChloroflexi mat on an iron mineral substrate. All other mats are on a siliceous sinter substrate

placed in a shallow Pyrex glass Petri dish, with examples illustrated in Fig. 1. The polarization spectra of the pure cultures were acquired in both a reflection and transmission mode and they were normalized to a reference white light spectrum obtained by using the same illumination source as the samples, an adjustable $150 \mathrm{~W}$ stabilized quartz-halogen fiber optic illuminator (Dolan-Jenner DC950). The polarization spectra of the photosynthetic microbial mats were acquired in reflection mode. The polarization was corrected for the (small) baseline polarization of the white light. No significant polarization was introduced by the Petri dish. These two configurations are described in more detail in the work of Sparks et al. (2009a, 2009b). In the reflection mode, unpolarized light exiting an integrating sphere illuminates the sample from above and is reflected or scattered directly into the polarimeter, which views the sample from above with an unobscured optical path. In the transmission mode, the illumination source is directed to a white plaque beneath the sample, and the light from this white scattering surface then passes through the sample and, as in the reflection measurement, from the sample directly into the polarimeter. Except where explicitly stated, the reflection measurements used a black mat background beneath the sample so that the reflected light was dominated by scattering solely from the microbial sample. We measured a number of the samples at a range of optical depths, and we determined that the signal in both transmission and reflection was robust against such variations. Once an optical depth of order unity is obtained, the polarization signal strength is roughly constant (Sparks et al., 2009b).

\section{Results}

\subsection{Pigment and polarization spectra: pure cultures}

Schwieterman et al. (2018) presented the absorbance spectra and characteristic wavelengths of features in the spectra of pigments (their figure 9 and Table 1). Figure 3 presents similar spectroscopic and polarimetric data for the filamentous anoxygenic phototroph (FAP) C. aurantiacus showing the circular polarization transmission and reflection spectra, the absorbance of the pigments in the intact cells measured by the polarimeter, and the in vivo spectrum of the pigment-protein complexes isolated from the cells, which was measured independently. The polarimeter absorbance spectrum is included to enable identification of pigment absorption maxima and correlate them to the pigment polarization features. The polarimeter generates clear absorption spectra while in transmission mode but yields less structured spectra while in reflection mode. Therefore, we measured the in vivo absorption spectra by using a different method and instrument to corroborate the polarimeter absorption measurements and in particular to help correlate 

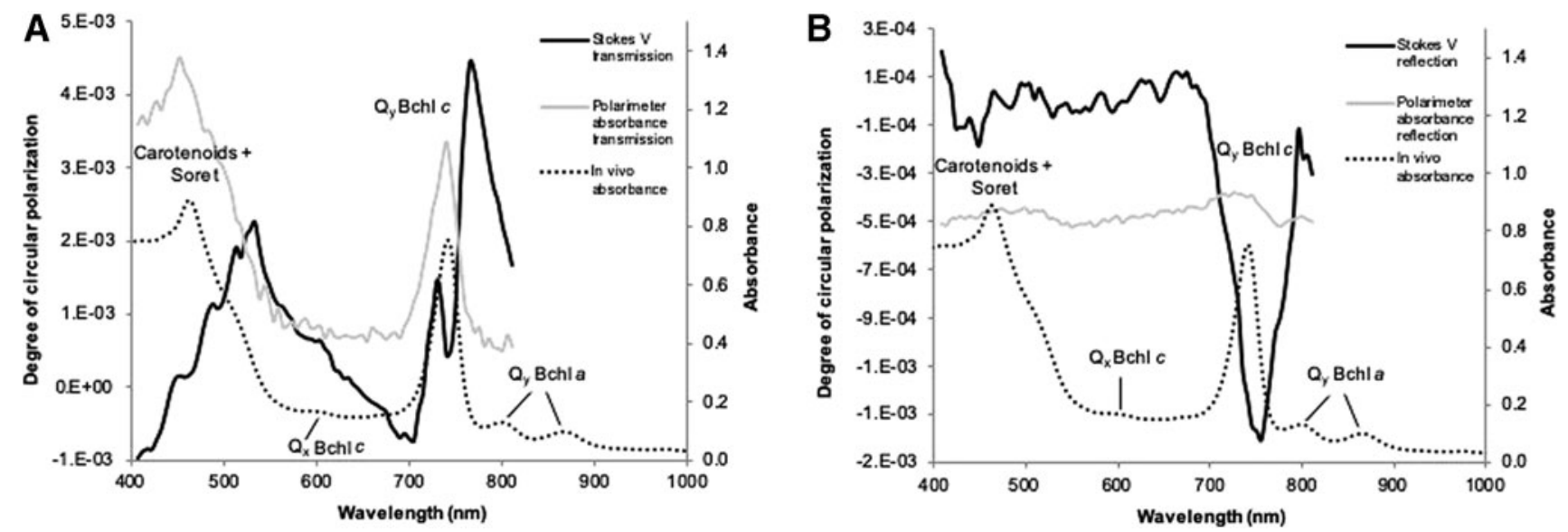

FIG. 3. Panel showing correlation between the in vivo absorbance, polarimeter absorbance, and degree of circular polarization (Stokes V) for the FAP Chloroflexus aurantiacus (A) in transmission and (B) in reflection. The alignment of the absorption maxima of the Qx and Qy bands of Bchl $c$ and the circular polarization signals are visible. Bchl, bacteriochlorophyll; FAP, filamentous anoxygenic phototroph.

and interpret pigment polarization features in reflection mode. This illustrates that (1) the absorption features of the whole cells measured by the polarimeter closely agree with the in vivo spectrum, and (2) the circular polarization features correlate with these absorption peaks. These are generic characteristics common to all samples, and the polarimeter absorption spectra are subsequently plotted in Figs. 4 and 5. Figure 3 identifies specific pigment absorption maxima and correlates them to polarization features, which recur in some or all of the other samples (see also Schwieterman et al., 2018).

Figures 4 and 5 present the results of the measurements for the pure cultures of the selected anoxygenic phototrophs, derived as described in the Section 2, with the upper row giving the transmission circular polarization spectra, the middle row the reflection circular polarization spectra, and the bottom row the absorbance spectra as measured by the polarimeter. The uncertainties shown with the data represent the standard deviations of the mean of multiple repeated measurements. Later, we discuss each of the samples in turn, roughly in decreasing order of the strength of the polarization signal.

3.1.1. C. aurantiacus. Figures $3 \mathrm{~A}, \mathrm{~B}$ and $4 \mathrm{a}, \mathrm{b}$ show the circular polarization spectra for transmission and reflection, respectively, for the pure culture of $C$. aurantiacus, an FAP. The transmission spectrum shows a large, broad polarization peak of $\sim 0.2 \%$ polarization at about $530 \mathrm{~nm}$. The shoulder of this prominent peak in the polarization spectrum may correspond to the $\mathrm{Q}_{\mathrm{x}}$ absorption band of $\mathrm{Bchl} c$, visible as a small peak in the absorption spectrum. The carotenoid absorption in the blue does not correspond to any strong polarization feature. At wavelengths longer than $700 \mathrm{~nm}$, there is a sharp rise in the circular polarization spectrum to a peak of $0.5 \%$, at about $760 \mathrm{~nm}$, with a dip on the rising slope at about $740 \mathrm{~nm}$, corresponding to the strong $\mathrm{Q}_{\mathrm{y}}$ absorption band of Bchl $c$. In the reflection polarization spectrum, the $\mathrm{Q}_{\mathrm{x}}$ features are absent. However, there is a very large, clean negative circular polarization feature, extending from 700 to $800 \mathrm{~nm}$, with a peak (absolute) value also at about $740 \mathrm{~nm}$, which is, within the uncertainties, at the location of the Bchl $c$ absorption maximum as shown in Fig. 4c. In the blue part of the spectrum, there are indications of additional weak polarization structure.

3.1.2. Rhodobacter capsulatus. Figure $4 \mathrm{~d}$ and e show the transmission and reflection polarization spectra of $R$. capsulatus, a purple non-sulfur anoxygenic phototroph. The main pigment utilized by this organism is $\mathrm{Bchl} a$, which has absorption peaks at about 805 and $860 \mathrm{~nm}$, and there is a monosignate negative polarization feature in the transmission spectrum at about $800 \mathrm{~nm}$. There is also a relatively strong polarization peak at about $510 \mathrm{~nm}$, potentially associated with carotenoids with polarization levels $\sim 0.1 \%$. By contrast, these features are absent from the reflection spectrum, where the significant (although weak) polarization peak is at an inconspicuous region of the transmission spectrum from 600 to $700 \mathrm{~nm}$. The in vivo pigment absorbance spectrum (not shown) has a small maximum at $675 \mathrm{~nm}$, but this peak is absent in the polarimeter-based absorption spectra shown in Fig. 4f.

3.1.3. C. tepidum. Figure $4 \mathrm{~g}$ and $\mathrm{h}$ show the transmission and reflection polarization spectra of $C$. tepidum, a green sulfur anoxygenic phototroph. In transmission, there is an extremely prominent antisymmetric polarization feature from 700 to $800 \mathrm{~nm}$, with a sign reversal at about $750 \mathrm{~nm}$, coinciding with the maximum $\mathrm{Q}_{\mathrm{y}}$ absorption of Bchl $c$, visible in Fig. 4i. The maximum degree of polarization for this highly distinctive profile is about $0.25 \%$. In the blue region of the spectrum, carotenoids or the Soret band of Bchl $c$ cause additional polarization structure. By contrast, in reflection, the overall polarization level is much reduced. The characteristic S-shaped curve of the transmission spectrum is absent, with instead, broad, weak polarization peaks to the red and to the blue. We also analyzed $C$. tepidum after subjecting the culture to freeze-thaw and lysis of the cells and release of chlorosomes and pigments, which show that the polarization increases by an order of magnitude (see 3.3 below for a full discussion). 


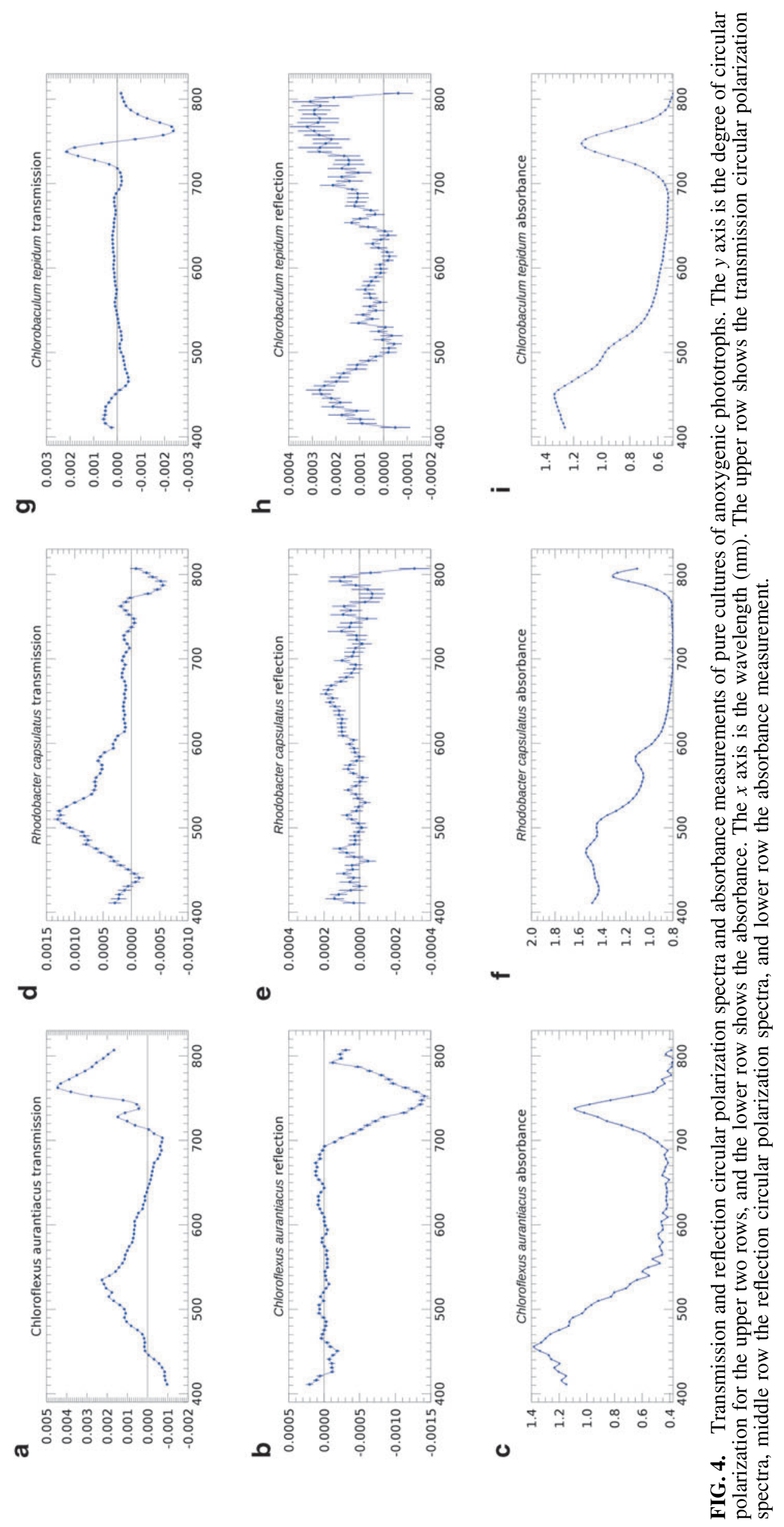




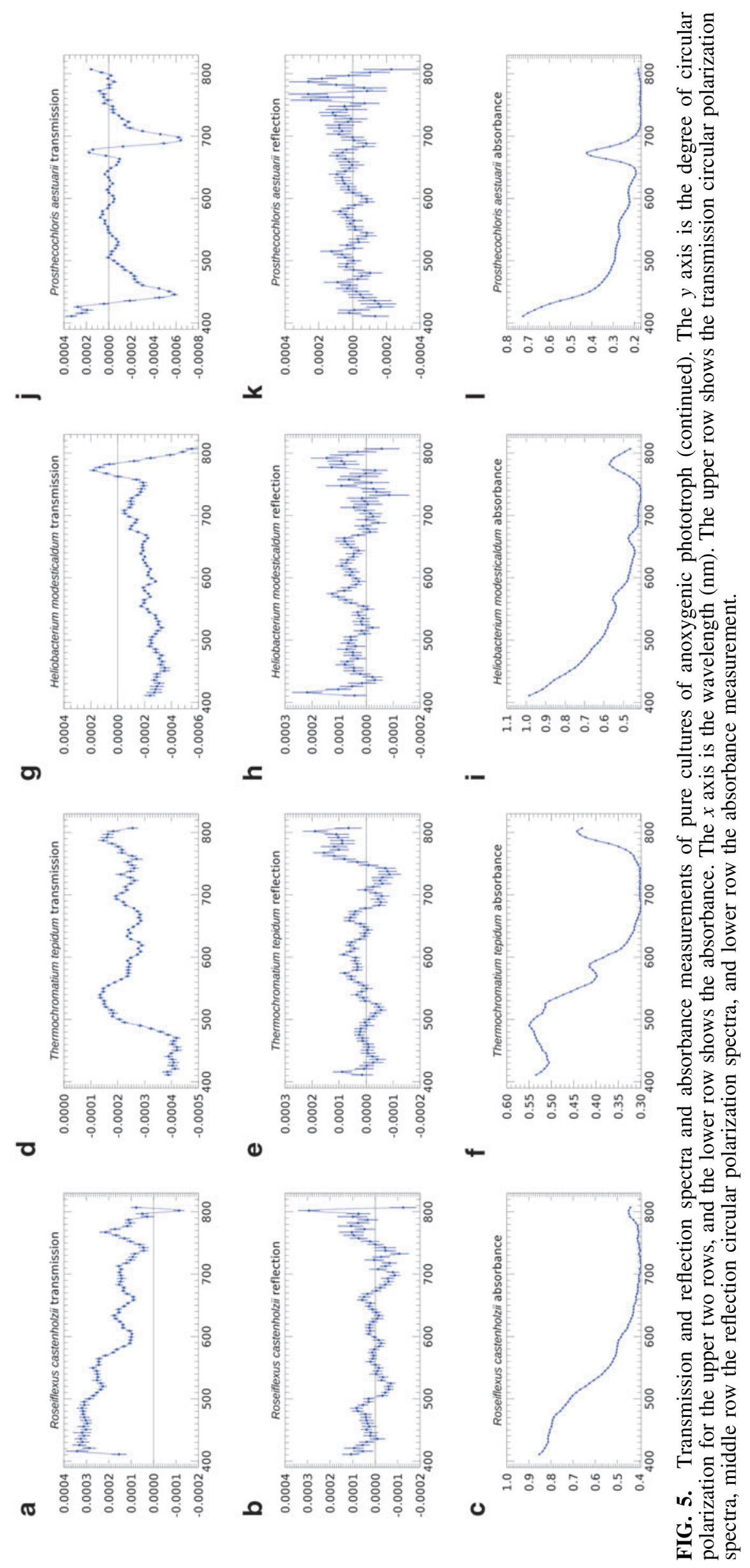


3.1.4. Roseiflexus castenholzii. Figure 5a and b show the transmission and reflection polarization spectra for $R$. castenholzii, another FAP. In contrast to Chloroflexus, the overall level of polarization is very weak, reaching at most $0.04 \%$. The in vivo absorption spectrum (not shown) reveals absorption of Bchl $a$ at 810 and $885 \mathrm{~nm}$, which is mostly beyond the range of this polarimeter. However, the absorption maxima at about $810 \mathrm{~nm}$, shown in Fig. 5c appears to be introducing rapid changes to the polarization spectrum in both transmission and reflection. Elsewhere, there are small peaks and dips in the polarization degree, potentially correlating with the $\mathrm{Q}_{\mathrm{x}}$ band of $\mathrm{Bchl} a$ and/or carotenoids.

3.1.5. Thermochromatium tepidum. Figure $5 \mathrm{~d}$ and e show the polarization spectra for $T$. tepidum, a purple sulfur anoxygenic phototroph, and the absorbance spectrum is shown in Fig. 5f. The transmission polarization spectrum is unusual in being entirely negative, with a flat, broad (negative) peak in polarization degree blueward of about $480 \mathrm{~nm}$. This may be another example of a broad S-shaped feature, from about $550 \mathrm{~nm}$ to the blue, corresponding to the prominent broad carotenoid absorption band. Additional polarization structure at about $800 \mathrm{~nm}$ is likely caused by the onset of the Bchl $a$ bands, which continue into the near NIR. In reflection, the primary feature is also toward the NIR part of the spectrum, although its amplitude is low.

3.1.6. Heliobacterium modesticaldum. Figure $5 \mathrm{~g}$ and $\mathrm{h}$ show the transmission and reflection polarization spectra for H. modesticaldum, a heliobacterium. In the transmission spectrum, a sharp peak around $780 \mathrm{~nm}$ is followed by a sign reversal and decrease continuing to the red edge of the detector range of sensitivity. The principal pigment associated with this organism is Bchl $g$, which has $\mathrm{Q}_{\mathrm{y}}$ absorbance maxima at about 674 and $788 \mathrm{~nm}$, shown in Fig. 5i, where polarization structure is evident, particularly at the red extreme of the spectrum. There is additional weak polarization structure across the entire spectrum in transmission. In reflection, only very weak polarization signatures are seen, with a weak feature at $570 \mathrm{~nm}$ which corresponds to the $\mathrm{Q}_{\mathrm{x}}$ absorbance band as shown in Fig. 5i. A sharp rise at the blue edge of the spectrum at about $400 \mathrm{~nm}$ appears to be significant.

3.1.7. Prosthecochloris aestuarii. Figures $5 \mathrm{j}$ and $\mathrm{k}$ show the polarization spectra for $P$. aestuarii, also a green sulfur anoxygenic phototroph. In transmission, a sharp negative peak can be observed at $445 \mathrm{~nm}$ and a clear S-shaped feature is present around $685 \mathrm{~nm}$. The polarization level for the reflected samples is very weak, and largely featureless. Interestingly, even though the microbe utilizes the pigments Bchl $c, a$ and Chl $a$ (Table 1), there is only a strong Chl $a$ absorption band present at about $680 \mathrm{~nm}$ (Fig. 51); with a corresponding polarization feature (Fig. $5 \mathrm{j}$ ).

\subsection{Pigment and polarization spectra: photosynthetic microbial mats}

Table 2 lists the major phototrophs and pigments present in the six photosynthetic microbial mats. Each mat typically contains two to three major photosynthetic groups, but these are complex communities containing thousands of species of microbes. Parenteau et al. (in preparation) will present reflection spectra of field samples in depth. Figure 6 shows a first attempt to ascertain the circular polarization levels from light scattered by such photosynthetic mats. In general, most mats did not yield a strong reflectance signal; however the Chocolate Pots Synechococcus-Chloroflexi mat, Figs. 6e and 7c, shows a significant and distinctive polarization maximum at $740 \mathrm{~nm}$, which corresponds to the absorption maximum of Bchl $c$ in Chloroflexus. Although this feature is clearly associated with $\mathrm{Bchl} c$, the sign is reversed relative to the pure culture. Such a sign reversal can occur at a specular reflection, though it is unclear where such a reflection might be occurring, or if we may be seeing a polarization-sensitive surface scattering/reflection phenomenon. The relative contributions of specularly reflected light and internally scattered light (where polarization can selfcancel) must differ between the pure culture and the more natural environment of the mat. There are other polarization structures within the spectra, at levels within the range of our precision polarimeter, and almost certainly associated with the pigmentation of the microbes in the mats, however in an absolute sense these polarization levels are low.

In addition, signals from membrane assemblies, such as the different architectures of intracytoplasmic membranes in purple bacteria, and chlorosomes in green sulfur and FAPs, can yield mixtures of signal shapes and signs simultaneously resulting from different levels of structural organization.

We have presented polarization spectra of pure cultures and of microbial mat communities that include some of the species discussed individually earlier. To analyze the three perspectives in combination, Fig. 7 displays a pure culture's (C. aurantiacus) transmission and reflection, which may be considered as end-member spectra contributing to the reflection of a microbial mat that contains a relatively high fraction of these phototrophs, and an example of such a mat. We observe a feature at $740 \mathrm{~nm}$ in the pure culture's reflected polarization spectrum. The same feature occurs with the sign reversed in both the pure culture's transmission and the microbial mat's reflection. Hence, we are able to trace a spectral polarization feature in an environmental sample to a specific biological source through the pure culture's endmember spectra. This ability to validate the biological nature of circular polarization signature-and techniques to deconvolve environmental spectra-will be significant for life detection where the biosignature may arise from complex communities.

\subsection{Enhancement of circular polarization signal upon cell lysis}

In our earlier work, we found that the reflection and transmission spectra of cyanobacteria were very closely related (Sparks et al., 2009a, 2009b). In contrast, the measurements in this study reveal significant differences in the two types of spectra. We, therefore, selected an extreme example of the discrepancy, as given by $C$. tepidum, contrasting Fig. $4 \mathrm{~g}$ and $\mathrm{h}$, for further study. The striking $\mathrm{S}$-shaped or derivative curve of the transmission spectrum, Fig. 4g, was not evident in the reflection experiment, Fig. 4h, in stark contrast to the cyanobacteria results of Sparks et al. (2009a). Hence, we obtained additional samples of C. tepidum cultures for this study (the "Jan16" 


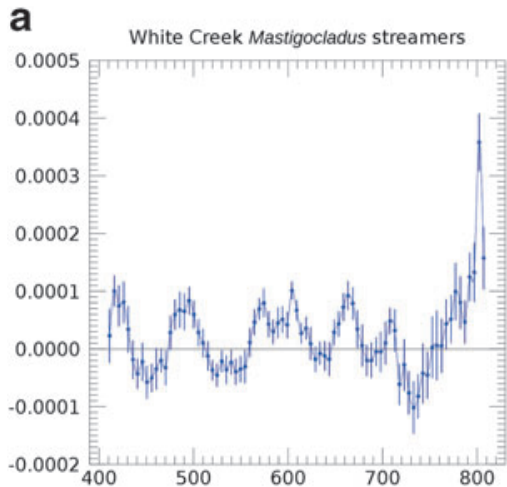

b

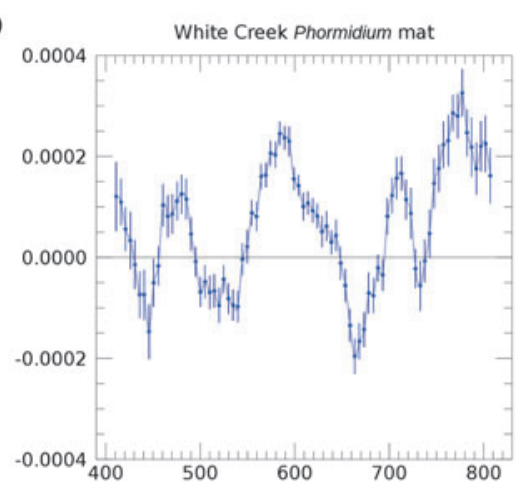

d

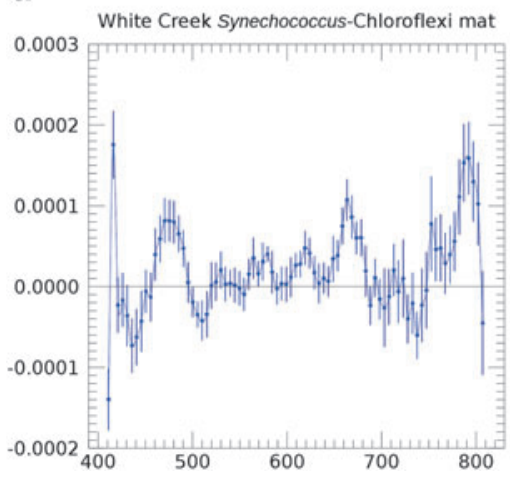

e

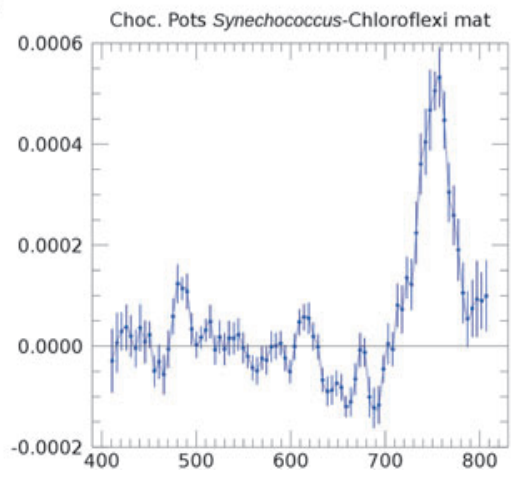

C

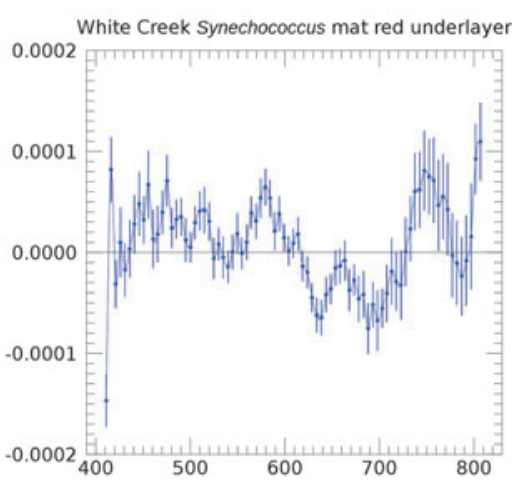

f

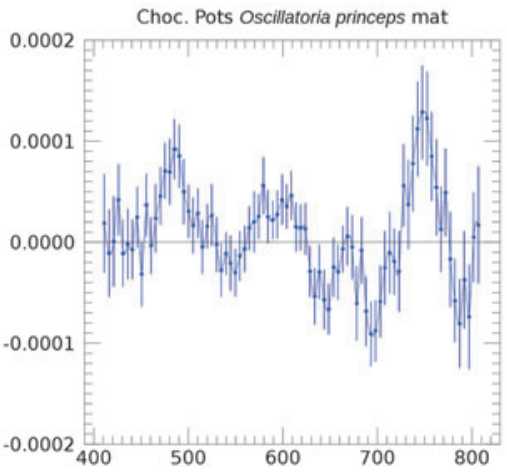

FIG. 6. Reflection circular polarization spectra of environmental photosynthetic microbial mats. The $y$ axis is the degree of circular polarization, while the $x$ axis is the wavelength (nm).

sample) but were unable to measure their polarization spectra for $\sim 4$ months due to a hardware failure. During this period, the samples were stored in a freezer at NIST and when thawed out, demonstrated clear indications of cell lysis and release of pigments into the media (see Section 2.1).

Figure 8a and $\mathrm{b}$ show the polarization spectra obtained in March 2015 for the original fresh culture, and the measurements obtained in May 2016 for the Jan 16 culture after a 4-month storage period at $-20^{\circ} \mathrm{C}$ (Fig. $8 \mathrm{c}$ and d). Figure 8e and $f$ show polarization spectra of the January 2016 sample obtained a full 2 years after the initial measurement (storage $-20^{\circ} \mathrm{C}$ ), obtained February 2018.
Figure 8a and $\mathrm{b}$ replot the data of Fig. $4 \mathrm{~g}$ and $\mathrm{h}$, now on the same scale. The transmission polarization spectrum of C. tepidum is extremely distinctive (especially the reversedsign feature straddling $750 \mathrm{~nm}$ corresponding to Bchl c). Despite this, the original reflection spectrum of the presumably healthy pure culture showed almost no polarization apart from a weak, low-level rise seen expanded in Fig. 4h. In 2016, the transmission spectrum showed a substantial change in the strength of the positive side of the double polarization feature: The hitherto positive side of the reversed-sign polarization feature (to the blue of $750 \mathrm{~nm}$ ) became negative, whereas the negative side (to the red) did a

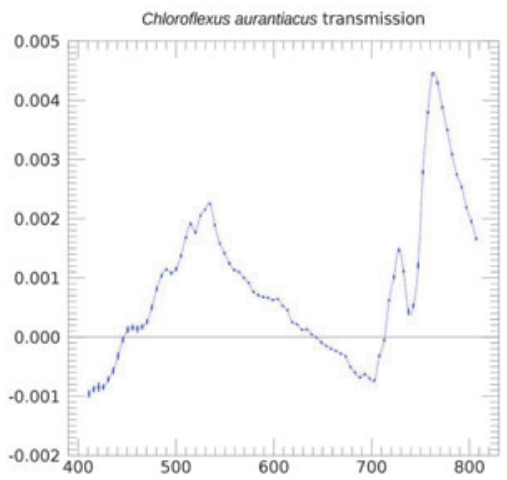

b

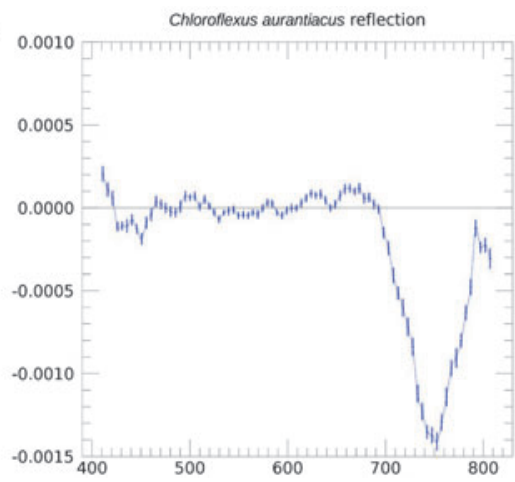

C

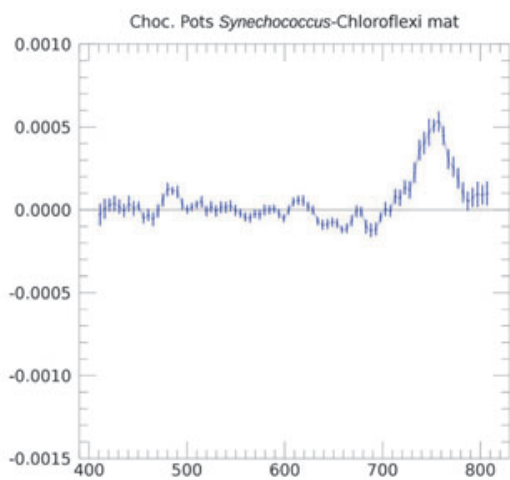

FIG. 7. The (a) transmission and (b) reflection circular polarization spectra of the pure culture Chloroflexus auranticus, which serve as end-member spectra for helping to deconvolve the (c) circular polarization spectra of the complex community in the Synechococcus-Chloroflexi photosynthetic mat from Chocolate Pots hot springs. The $y$ axis is the degree of circular polarization, whereas the $x$ axis is the wavelength $(\mathrm{nm})$. 

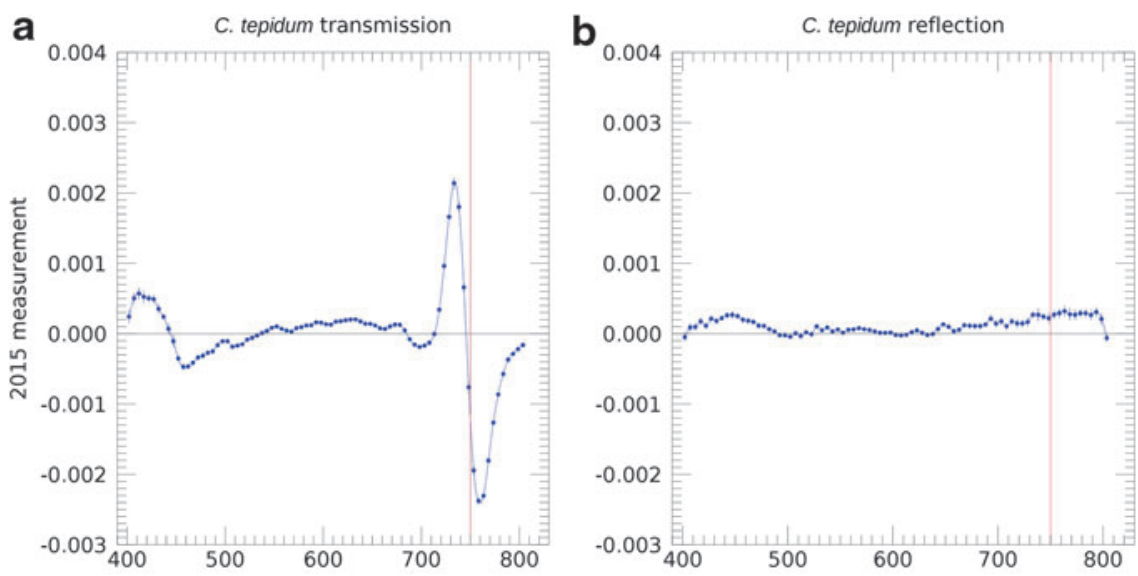

FIG. 8. Time course measurements revealing change in the transmission and reflection circular polarization spectra of the green sulfur anoxygenic phototroph Chlorobaculum tepidum. Surprisingly, the reflection spectrum of the $C$. tepidum culture was strengthened as the cells lysed and the chlorosomes containing the Bchl $c$ pigments were released $(\mathbf{b}, \mathbf{d}, \mathbf{f})$. The $y$ axis is the degree of circular polarization, and the red (vertical) line indicates $750 \mathrm{~nm}$, the wavelength of the Qy peak of Bchl $c$ in vivo, which can occur in the range $740-755 \mathrm{~nm}$.

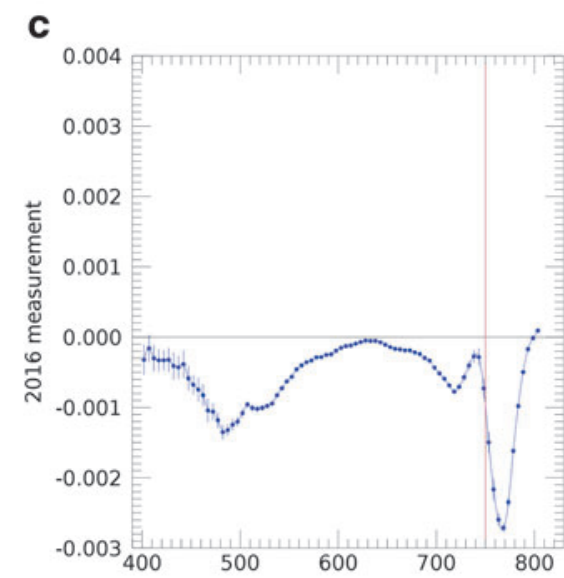

d
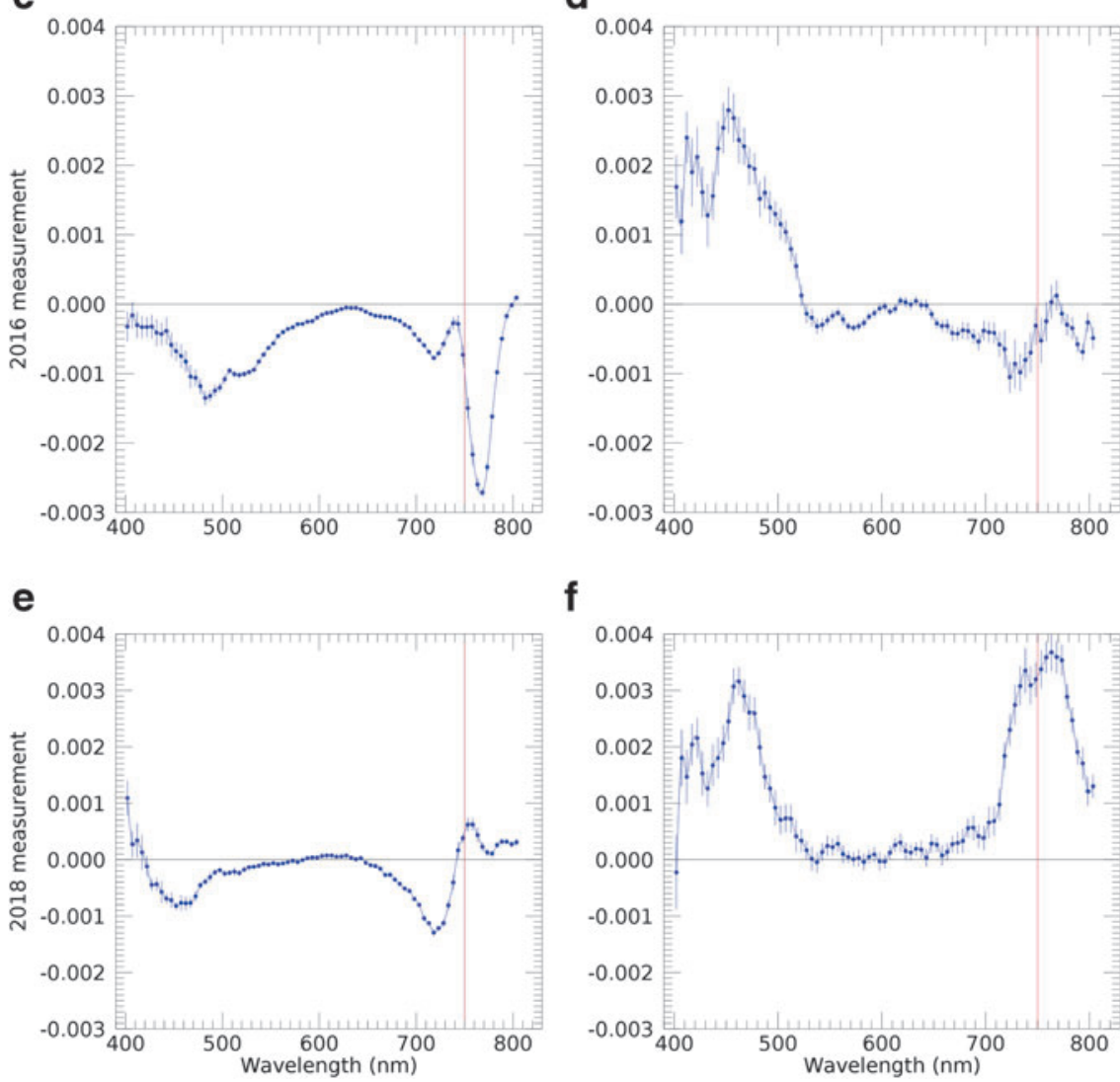

$\mathbf{f}$

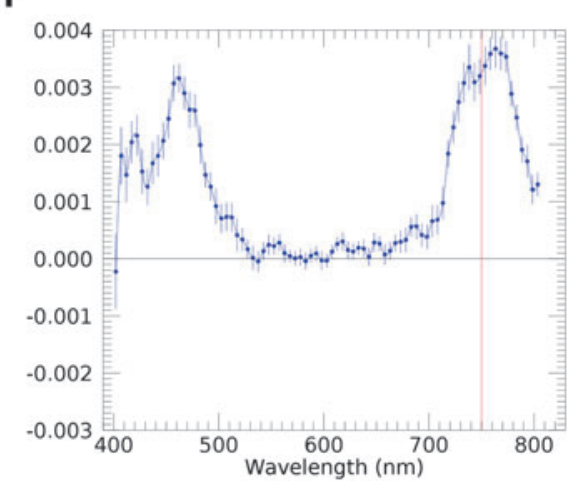

not show much change initially, before weakening. This is reminiscent of the type of changes observed in decaying leaf matter (Patty et al., 2017), where the negative and positive chlorophyll bands evolve independently. Interestingly, at this time, the reflection spectrum from the lysed $C$. tepidum sample showed a very strong polarization feature from 400 to $500 \mathrm{~nm}$ (corresponding to the Soret absorption band of Bchl $c$ ), about an order of magnitude amplification relative to the previously existing weak feature of the healthy culture. In 2018, after 2 years of storage at $-20^{\circ} \mathrm{C}$ and further cell lysis, the transmission spectrum continued its evolution; the polarization feature from 750 to $800 \mathrm{~nm}$ decreased in amplitude and reversed sign. Meanwhile, the reflection spectrum developed a second extremely prominent polarization feature from about $720-800 \mathrm{~nm}$ at the same wave- lengths as the strong polarization feature in the transmission experiment, which is associated with the $\mathrm{Q}_{\mathrm{y}}$ transition of Bchl c. Again, this is an increase of about an order of magnitude compared with the intact, healthy sample. We discuss reasons why this remarkable enhancement of polarization may have occurred in Section 4.

\section{Discussion}

\subsection{Spectropolarimetry of pure cultures and photosynthetic mats}

We have undertaken a systematic study characterizing the circular polarization spectra of pure cultures of anoxygenic phototrophs, and we used these spectra to help deconvolve the spectra of complex photosynthetic microbial 
mat communities in the natural environment. We began with measuring the in vivo absorption spectra of isolated pigments and pigment-protein complexes from each culture, and we used those data to help interpret the circular polarization transmission and reflectance spectra of the cultures, and then the microbial mats. Knowing the characteristics of the constituent pigments enables us to identify features in pure microbial cultures, and knowing the features of individual microbial species allows us, in principle, to understand the composite spectra of microbial mat communities. This was demonstrated in Fig. 7, where we were able to trace the Bchl $c$ present in the pure culture of Chloroflexus into the complex environmental Synechococcus-Chloroflexi photosynthetic mat.

Sparks et al. (2009a, 2009b) presented the first extensive study on oxygenic photosynthetic microbes from a similar (astronomical) perspective, in which it was found that the polarization spectra of a pure culture of cyanobacteria in reflection mimicked those in transmission, apart from the squared absorption amplitude resulting from the double pass through the culture. The polarization features correlated well with the spectral features, and both monosignate and derivative-shaped curves were found to be associated with the Chl $a$ pigment and accessory pigments phycocyanin and phycoerythrin. From those initial cyanobacteria pure culture results, a simple heuristic explanation of the relationship between polarization in reflection and transmission was presented, namely that optical depth dominated in both cases. It was supposed that for a reflection experiment, the incoming light beam penetrated to a multiple scattering surface defined by unit optical depth, below which polarization information was lost, but that by necessity it reemerged through the layer between unit optical depth and zero optical depth in a fashion analogous to the transmission experiment, resulting in similar polarization spectra.

Here, in a second major study of microbes encompassing a more diverse suite of pigments, phototrophs, and environmental microbial mats, we find an extraordinary range of polarization properties and interrelationships between reflection, transmission, and absorption. A conclusion of this study is that there remains a great deal more to understand and learn than the early indications from the cyanobacteria pure culture led us to believe.

Unlike the previously studied pure cultures, here we found little correspondence between the reflected and transmitted spectra. In some cases, the polarization spectra were completely different, and in others, features from one pigment were present in both, whereas other pigments were not, challenging the task of global prediction of signal strength from, for example, the CD literature. There were transmission features absent in the reflection spectra, though conversely, only a small number of features in the reflection spectra did not appear in the transmission spectra-the unidentified reflection feature from $600 \mathrm{~nm}$ to $650 \mathrm{~nm}$ in $R$. capsulatus and the spike at the blue edge of the $H$. modesticaldum spectrum. Interestingly, in some cases, the features are present in both reflection and transmission spectra, but with large differences in signal magnitude between them. Some of these differences are well illustrated by the spectra of $C$. aurantiacus, Fig. 7, which seems to have many more features identifiable in transmission than in reflection. In reflection, a single strong band dominates, with shoulders at 730 and $765 \mathrm{~nm}$, apparently correlating with features present in transmission.

Nevertheless, we are able to identify individual biological elements within complex natural microbial communities, and to potentially deconvolve environmental spectra, including polarization, into their constituents.

The cause of these differences remains unresolved, with candidate effects ranging from the internal cell structure and arrangement of photosynthetic apparatus, to macroscopic scattering phenomena external to the microbes. A polarization sign reversal on reflection could, in principle, null the dichroic effect of the medium on the beam as it propagates back through the sample. Other factors could relate to the geometry of the pigments relative to the cells and their membranes or internal cell structure (e.g., pigments arranged in chlorosomes and chiral domain organization, Patty et al., 2018), which we discuss later.

\subsection{Chirality and structural organization of pigment systems}

Historically, CD measurement of the photosynthetic apparatus of microbes has taken place on isolated fractions, and it is dependent on the surrounding chemical environment (including use of solvent), concentration, and temperature. Our circular polarization measurements were performed on whole cells and complex communities of microbes, which is more relevant for treatment of chirality as a surface biosignature for life detection. These polarization spectra represent a mixture of the features, signs, and magnitude associated with pigments and pigment complexes at several layers of structural organization in whole cells, as well as in multilayered photosynthetic mats. The pigments and the structural arrangements of the pigments vary considerably among the different photosynthetic microorganisms, and there appears to be a corresponding remarkable variety of circular polarization spectra. In addition, as noted earlier, polarization spectra are additive, and a variety of signals interact to yield net spectra for each experimental measurement. As such, this renders the interpretation of measurements on environmental samples more complicated. We have endeavored to identify wherever possible the biophysical reasons for the resultant spectra (noting that from a life detection perspective, this is not expected to be critical).

In general, there are three levels of structural organization that can generate CD signals: (1) Photosynthetic pigments are intrinsically chiral, as they are composed of asymmetric carbon atoms, (2) excitonic coupling between two or more pigment molecules (where the excited state is delocalized over a few pigments), and (3) larger macromolecular pigment-protein complexes and membrane assemblies (Garab and van Amerongen, 2009, and references therein). $\mathrm{CD}$ studies of monomeric solutions of chlorophyll reveal that the substituents at the asymmetric carbon numbers 10 , 8 , and 7 control the sign and magnitude of the spectra (Houssier and Sauer, 1970), which are generally believed to reflect the shape of the pigment absorption maxima. However, due to the planar geometry of photosynthetic pigments, these intrinsic signals are generally weak, and they cannot be identified in our transmission and reflection spectra. The signals arising from excitonic coupling are stronger, and they have a characteristic sinusoidal shape with positive and 
negative bands. The Bchl $c$ in $C$. tepidum provides an example of this in Fig. 4c. The signals from larger chiral polymer and salt-induced (psi-type) aggregates yield nonconservative anomalously shaped bands. These can extend beyond the molecular absorption bands (Keller and Bustamante, 1986; Garab and van Amerongen, 2009). In addition, signals from membrane assemblies, such as the different architectures of intracytoplasmic membranes in purple bacteria, and chlorosomes in green sulfur and FAPs, can yield mixtures of signal shapes and signs simultaneously resulting from different levels of organization.

The effect of the structural organization of the pigments on the shape and magnitude of the circular polarization is well illustrated by the course time measurements on C. tepidum (Fig. 8). Circular polarization measurements on chlorosomes generally show a large diversity, resulting from two overlapping CD spectra (Griebenow et al., 1991). Within the chlorosomes, the pigments are self-coordinated rather than being oriented by the pigment-protein environment. Likely, on lysis of the cells, the pigments have auto-assembled into aggregates larger than those in the chlorosomes, and the spectral shape in transmission corresponds with reported size-dependent changes in CD (Prokhorenko et al., 2003). It is more difficult to directly explain the much larger signal in reflection, similar to the observed large differences in the chlorosomes of $C$. aurantiacus. Other factors likely play a large role in the differences between transmission and reflection polarization measurements on chlorosomes.

It is highly likely that the transmission and reflection spectra contain mixtures of all signal types, and thus it is often hard to assign specific features to any particular structural organization. Nevertheless, what has emerged from this study is a catalog of biological circular polarization spectra that have no known abiotic mimics, whose magnitude (degree of circular polarization) is stronger than previously appreciated, which helps bolster the potential of circular polarization spectra as a biosignature.

\subsection{Relevance to life detection on Solar System bodies and exoplanets}

The majority of the photosynthetic microbial samples studied here exhibited degrees of polarization in transmission at levels up to $v \cong 0.5 \%\left(5 \times 10^{-3}\right)$. In reflection, the range is about $10^{-4}$ to $10^{-3}$, consistent with previous findings for cyanobacteria and other organisms studied: a modest signal, but still some two orders of magnitude higher than ambient abiotic polarimetric "noise." In Section 3.3, we discussed an empirically observed amplification of the signal that exceeds this range. Other studies have found additional examples of higher circular polarization levels (Patty et al., 2019), up to 2\%. It is essential to accumulate a broader understanding of the range of microbial polarization levels present, and empirical data such as Earth observations from space that include both microbial and vegetation dominated scenery would be invaluable for exoplanet surface biosignature modeling and experiment design based on remote detection of chiral signatures.

If a visible light or ultraviolet (UV) spectropolarimeter is included on a large telescope, it may be able to measure the linear and circular polarization of photosynthetic microbes, and characterize chiral biosignatures, even if the pigments differ substantially from those found on Earth. As noted in the introduction, photosynthetic microbes inhabiting continental areas, marine intertidal, and shallow marine settings on exoplanets can provide surface biosignatures for the remote detection of life (Schwieterman et al., 2018). The reflectance spectra of the photosynthetic pigments [e.g., the red edge of Chl $a$, or the NIR-edge of purple bacteria (Sanromá et al., 2014)] could be detectable through different cloud coverage levels and atmospheric compositions inferred via direct imaging by next-generation space telescopes, such as the Large Ultraviolet/Optical/Infrared Surveyor (LUVOIR), which is one of four Decadal Surveys on Astronomy and Astrophysics (Astro2020) Mission Concept Studies (LUVOIR Final Report, 2019)*.

Although the reflectance spectra of these features are generally weaker than the transmission spectra by an order of magnitude, they could be observed with roughly $10,000 \mathrm{~h}$ on a LUVOIR-size telescope with a visible light spectropolarimeter, in the case of an inner-edge habitable zone terrestrial super Earth $\left(\mathrm{R} \sim 1.6 \mathrm{R}_{\mathrm{E}}\right)$ covered in the pigment. Currently, the proposed LUVOIR-A architecture concept includes a UV wavelength high-resolution spectropolarimeter called POLLUX. Estimates for the UV spectropolarimeter POLLUX as described in the LUVOIR Final Report (LUVOIR Final Report, 2019) show that for the $15 \mathrm{~m}$ primary architecture A design, a parts-per-million level polarization would be detectable after only a few hours of integration time around bright, nearby $(<50 \mathrm{pc}$ [parsec]) stars. However, in polarimetry, we must consider both precision and the available flux and thus must scale the signal by the anticipated light reflected by the planet, either in combination with that of the star, or isolated from the star but much fainter. Hence, it is highly unlikely that the next envisaged generation of telescopes could apply these techniques directly to Earth-like planets around Solar-type stars.

A parts-per-million magnitude signal (like that of the pigments reflectance spectra shown in this study) could, in theory, be detected on a terrestrial super Earth with only $\sim 100 \mathrm{~h}$ of observations with a visible light spectropolarimeter if it orbited closely ( $\sim 0.05 \mathrm{AU})$ to the star; however, such planets are unlikely to harbor life for typical Solar-type stars. (Signal-to-noise ratio (SNR) limit estimates are not calculated for a visible light polarimeter for LUVOIR as none are currently planned, so our estimates are based roughly on the instrumental performance of top-performing groundbased telescopes, and the precision of POLLUX). It is worth noting that the UV Polarimeter POLLUX can reach SNR within instrumental limits for stars with $U$ band magnitudes brighter than $\mathrm{M} \sim 18$ after a few hours of integration time (see figure 13-5 in the LUVOIR Final Report) and that in general the SNR limits improve toward longer wavelengths.

Both the UV and visible regions of a spectrum, however, can provide chiral signatures. By utilizing the UV spectral region rather than the visible, there is potential access to a plethora of optically active transitions associated with biomolecules such as nucleic acids, proteins, and amino acids, including, for example, protein secondary structures, such as the $\alpha$-helix. For remote observation, the relative contributions of the star's spectral energy distribution and influence

\footnotetext{
*https://asd.gsfc.nasa.gov/luvoir/reports/ (as of 11/09/2020)
} 
of the presence of an atmosphere should be taken into consideration. Although cooler stars, in general, have lesser amounts of UV flux, this can be compensated for by (1) their habitable zone, which is closer to the star that improves an exoplanet's contrast ratio relative to the star; (2) many faint cool stars are extremely active, and exhibit brief, very luminous UV flares that can illuminate close-in habitable exoplanets. Light echoes of these flares by the exoplanet further improve the contrast ratio due to the time delay for the flare's arrival (Sparks et al., 2018). With a time-resolved instrumental capability, such a technique could be available to LUVOIR. Note that the use of light echoes allows access to planets that are very close to their host star, and this does not require a coronagraph.

There is an increasing appreciation of aerobiology, although in situ microbial communities inhabiting terrestrial aerosol particles and dust are not yet well established (Smith, 2013). The possibility of life in the clouds of Venus has been discussed for decades (Morowitz and Sagan, 1967 and many others subsequently). Seager et al. (2020) presents a detailed account of a potential Venusian aerial biosphere utilizing a droplet habitat and cyclical, dynamic life cycle (see also Greaves et al. 2020). Survival in this environment would prove extremely challenging given, for example, the exceedingly low water activity of the sulfuric acid droplets. Nevertheless, Venus offers a potential Solar System application for an empirical, chiral remote-sensing experiment. Conceivably, if an exoplanet's atmosphere were inhabited densely enough by microorganisms, transit spectropolarimetry might be a feasible detection method (Smith, 2013) identified by Strassmeier et al. (2014) as a possible Extremely Large Telescope key application. In that vein, Judge (2017) proposed spectropolarimetry of the vapor plumes of Europa and Enceladus as they transit Jupiter and Saturn, respectively, as a means to probe for the presence of biomarkers.

Within the Solar System in general, polarimetric methods lend themselves to remote-sensing surveys of targets seen in scattered light, such as the vents and deposition blankets of Ocean World plumes, whereas in situ instrumentation could utilize either transmissive or reflective configurations applied to locally harvested samples. Biological pigments can serve to protect against UV radiation (Cockell et al., 2003; Schwieterman et al., 2018), and, coupled to other survival strategies, may be utilized by exotic forms of extant life. Evidence may be sought for preserved chiral signatures in subsurface or otherwise-protected environments, whereas the remains of biological organisms can also retain a chiral signature after recent exposure to a hostile environment, such as ejection in a plume (Hand et al., 2009).

\section{Conclusions}

We have undertaken a systematic study of increasing complexity, from spectra of purified pigment samples, through pure cultures of intact phototrophs, and from there to complex microbial communities from the natural environment on different mineral/rock substrates. By doing so, we were able to understand the biophysical origin of the signals present in both the spectroscopic and polarimetric data, though differences between reflection and transmission remain unexplained. We found correlations between the polarimetric signatures arising from the chirality of the biomolecules and the corresponding absorption spectra. In addition, we encountered an unsuspected, enormously rich diversity in almost every characteristic. We found that, contrary to anecdotal assumptions, the circular polarization signal can be strong; that some but not all pigments exhibit a polarization signature; and that the reflection polarization spectra differ in, sometimes, major ways from the transmission spectra, unlike those of earlier cyanobacteria studies. The polarization signatures are correlated with spectral absorption features, as expected, and are comprehensible in the context of our knowledge of the implicit pigments and pigment complexes in the organisms, but in ways that differ from organism to organism and pigment to pigment.

Reflectance measurements of the circular polarization spectra of pure cultures and microbial mats from a variety of environmental settings, here and in earlier work, show that biological pigment absorption and circular polarization are strongly coupled. If photosynthesis were to evolve on a habitable terrestrial exoplanet, polarimetric measurements could help interpret spectral data from a future life detection mission, and allow us to infer a biological presence even if pigmented phototrophs evolved to have different absorption maxima given the different stellar spectral irradiance and different atmospheric composition.

We discovered that one of the studied cultures, chosen because its transmission and reflection polarization spectra differed, exhibited an increase in polarization signal strength in reflected light by an order of magnitude as the culture degraded in health and evidence of cell lysis became apparent. For individual measurements, the reflected signal reached between $0.5 \%$ and $1 \%$, far higher than the canonical $10^{-3}$ polarization level characteristic of the reflected light polarization of cyanobacteria (Sparks et al., 2009a). Patty et al. (2019) found in their study of eukaryotic phototrophic organisms, that samples of brown algae Fucus serratus and Fucus spiralis showed an extremely high degree of circular polarization in transmission, up to $2 \%$ polarization, whereas other algae in their study showed weaker or no signals, which corresponded to a range in polarization properties within the algae of a factor of 1000 ! We conclude that: (1) the anecdotal "very low signal strength" for polarization in life detection does not always hold and, in fact, the signals can be very strong, and (2) strong polarization signals can persist after the destruction of the microbial samples.

Hence, in the anoxygenic phototroph samples, the signal is present not only for extant, living, healthy microbial communities but also in decaying cultures with cells breaking apart and, presumably, releasing their pigments into the ambient medium. We do not know how long the dramatic signal enhancement that resulted will persist. In our experiments, it remained high, and it was continuing to increase after 2 years.

The addition of polarimetric information adds a unique and crucial element to the life-detection endeavor, namely, an ability to infer with remote sensing the presence of chirality, one of the most important, pure biosignatures. In an exoplanet context, photosynthesis and, in particular, anoxygenic photosynthesis have been present for most of the history of terrestrial life, and given its potential to achieve global significance, may reasonably be expected to arise elsewhere and provide a potential biosignature visible for that entire history. The techniques are also applicable for exploration within the Solar System, where there are 
plentiful photons. The anticipated absence of photosynthesis elsewhere in the Solar System suggests targeting the UV spectral domain where strong chiral biological signals abound arising from generic biological material such as amino acids and protein secondary structures, such as the $\alpha$-helix. From an astronomical perspective, the mere presence of a polarization signal is evidence of the presence of chirality and hence life, though from a biological perspective it is important to dig deeper into the underlying biophysics and biochemistry. By doing so, we gain not only knowledge of the hypothetical exospecies but also insight into their biology.

We conclude that we have hit on a truly remarkable multiplicity of empirical results, defying systematic consolidation, implying that the study of circular polarization as a biosignature is in its infancy. Much more needs to be done to tease out the underlying systematics and causal influences. Empirically, however, the mere presence of circular polarization signatures, sometimes strong, associated with chiral biomolecules bodes well for its use, both in situ and in remote sensing, as an important universal, agnostic biosignature.

\section{Disclaimer}

Certain commercial equipment, instruments, or materials are identified in this article to specify the experimental procedure adequately. Such identification is not intended to imply recommendation or endorsement by the authors' organizations, nor is it intended to imply that the materials or equipment identified are necessarily the best available for the purpose.

\section{Author Disclosure Statement}

No competing financial interests exist.

\section{Funding Information}

This work was performed by members of NASA's Virtual Planetary Laboratory, a team in the Nexus for Exoplanet Systems Science (NExSS) research coordination network, and supported by the National Aeronautics and Space Administration under Cooperative Agreement Number NNA13AA93A, and grant number 80NSSC18K0829.

\section{References}

Blankenship RE (2014) Molecular Mechanisms of Photosynthesis, $2^{\text {nd }}$ ed., Wiley, UK \& USA.

Bryant DA, Costas AMG, Maresca JA, et al. (2007) Candidatus Chloracidobacterium thermophilum: an aerobic phototrophic acidobacterium. Science 317:523-526.

Cockell CS, Rettberg P, Horneck G, et al. (2003) Measurements of microbial protection from ultraviolet radiation in polar terrestrial microhabitats. Polar Biol 26:62-69.

Des Marais DJ, Nuth J, Allamandola LJ, et al. (2008) The NASA Astrobiology Roadmap. Astrobiology 8:715-730.

Garab G, van Amerongen H (2009) Linear dichroism and circular dichroism in photosynthesis research. Photosynth Res 101:135-146.

Glavin DP, Burton EJ, Elsila JE, et al. (2020) The search for chiral asymmetry as a potential biosignature in our solar system. Chem Rev 120: 4660-4689.

Goesmann F, Rosenbauer H, Bredehöft JH, et al. (2015) Organic compounds on comet 67P/Churyumov-Gerasimenko revealed by COSAC mass spectrometry. Science 349:aab0689.
Goesmann F, Brinckerhoff WB, Raulin F, et al. (2017) The mars organic molecule analyzer (MOMA) instrument: characterization of organic material in martian sediments. Astrobiology 17:655-685.

Greaves J, Richards AMS, Bains W, et al. (2020) Nature Astronomy, Sept 2020; doi: 10.1038/s41550-020-1174-4.

Griebenow K, Holzwarth AR, van Mourik F, et al. (1991) Pigment organization and energy transfer in green bacteria. 2 . Circular and linear dichroism spectra of protein-containing and protein-free chlorosomes isolated from Chloroflexus aurantiacus strain Ok-70-fl. Biochim Biophysica Acta Bioenerg 1058:194-202.

Hand KP, Chyba CF, Priscu JC, et al. (2009) Astrobiology and the potential for life on Europa. In Europa, edited by $\mathrm{R}$ Pappalardo, W McKinnon, and K Khurana, University of AZ Press, Tucson, AZ, pp 599-629.

Houssier C, Sauer K. (1970) Circular dichroism and magnetic circular dichroism of the chlorophyll and protochlorophyll pigments. J Amer Chem Soc 92:779-791.

Judge P (2017) A novel strategy to seek biosignatures at Enceladus and Europa. Astrobiology 17:852-861.

Keller D and Bustamante C. (1986) Theory of the interaction of light with large inhomogeneous molecular aggregates. II. Psitype circular dichroism. J Chem Phys 84:2972-2980.

Kelly SM and Price NC (2000) The use of circular dichroism in the investigation of protein structure and function. Curr Protein Peptide Sci 1:349-384.

MacDermott AJ (1998) SEXSOH: the search for EXtra-SOlar homochirality. In Proceedings of the IX Rencontres de Blois 1997, edited by LM Celnikier and JT van Than, presumably, Editions Frontieres, France, pp 522-529.

MacDermott AJ (2012) Perspective and concepts: biomolecular significance of homochirality: the origin of the homochiral signature of life. In Comprehensive Chirality, Vol. 8, edited by EM Carreira and $\mathrm{H}$ Yamamoto, Elsevier, Amsterdam, pp 11-38.

McKay CP, Grunthaner FJ, Lane AL, et al. (1998) The Mars Oxidant experiment (MOx) for Mars '96. Planet Space Sci 46:769-777.

Meadows VS, Reinhard CT, Arney GN, et al. (2018) Exoplanet biosignatures: understanding oxygen as a biosignature in the context of its environment. Astrobiology 18:630-662.

Morowitz H and Sagan C (1967) Life in the clouds of Venus? Nature 215:1259-1260.

Neveu M, Hays LE, Voytek MA, et al. (2018) The ladder of life detection. Astrobiology 18:1375-1402.

Patty CHL, Visser LJJ, Ariese F, et al. (2017) Circular spectropolarimetric sensing of chiral photosystems in decaying leaves. JQSRT 189:303-311.

Patty CHL, ten Kate IL, Sparks WB, et al. (2018) Remote sensing of homochirality: a proxy for the detection of extraterrestrial life. In Chiral Analysis: Advances in Spectroscopy, Chromatgraphy and Emerging Methods, $2^{\text {nd }}$ ed., edited by PL Polavarapu, Elsevier, Amsterdam, Netherlands, p 29.

Patty CHL, Ariese F, Buma WJ, et al. (2019) Circular spectropolarimetric sensing of higher plant and algal chloroplast structural variations. Photosynth Res 140:129-139.

Pierson BK and Castenholz RW (1974) Studies of pigments and growth in Chloroflexus aurantiacus, a phototrophic filamentous bacterium. Arch Microbiol 100:283-305.

Pierson BK and Parenteau MN (2000) Phototrophs in high iron microbial mats: microstructure of mats in iron-depositing hot springs. FEMS Microbiol Ecol 32:181-196.

Prokhorenko VI, Steensgaard DB, Holzwarth AR (2003) Exciton theory for supramolecular chlorosomal aggregates: 1 . 
aggregate size dependence of the linear spectra. Biophys $J$ 85: 3173-3186.

Rossi L and Stam DM (2018) Circular polarization signals of cloudy (exo)planets. $A \& A$ 616:A117.

Sanromá E, Pallé E, Parenteau MN, et al. (2014) Characterizing the Purple Earth: modeling the globally integrated spectral variability of the Archean Earth. ApJ 780:11.

Schwieterman EW, Kiang NY, Parenteau MN, et al. (2018) Exoplanet biosignatures: a review of remotely detectable signs of life. Astrobiology 18:663-708.

Seager S, Petkowski JJ, Gao P, et al. (2020) The Venusian lower atmosphere haze as a depot for desiccated microbial life: a proposed life cycle for persistence of the Venusian aerial biosphere. Astrobiology 21. DOI: 10.1089/ast.2020.2244.

Senge MO, Ryan AA, Letchford KA, et al. (2014) Chlorophylls, symmetry, chirality, and photosynthesis. Symmetry 6:781-843.

Smith DJ. (2013) Microbes in the upper atmosphere and unique opportunities for astrobiology research. Astrobiology 13:981-990.

Sparks WB, Hough J, Germer TA, et al. (2009a) Detection of circular polarization in light scattered from photosynthetic microbes. Proc Natl Acad Sci U S A 106:7816-7821.

Sparks WB, Hough JH, Kolokolova L, et al. (2009b) Circular polarization in scattered light as a possible biomarker. JQSRT 110:1771-1779.

Sparks WB, White RL, Lupu RE, et al. (2018) The direct detection and characterization of M-dwarf planets using light echoes. ApJ 854:134.

Strassmeier KG, Carroll TA, Mallon M (2014) Biosignatures for circular spectropolarimetry: key science for ELTs? In Search for Life Beyond the Solar System. Exoplanets, Biosignatures \& Instruments. Available online at https://astrobiology.nasa.gov/ nai/seminars/featured-seminar-channels/conferences-andworkshops/2014/3/16/search-for-life-beyond-the-solar-systemconference/index.html (accessed November 11, 2020).

Wald G (1957) The origin of optical activity. Ann N Y Acad Sci 69:352-368.

Address correspondence to: William B. Sparks SETI Institute

189 N Bernardo Ave \#200 Mountain View CA 94043 USA

E-mail: wsparks@seti.org

Submitted 6 April 2020

Accepted 12 October 2020

Associate Editor: Christopher McKay

$\begin{aligned} & \text { Abbreviations Used } \\ \mathrm{Bchl} & =\text { bacteriochlorophyll } \\ \mathrm{CD} & =\text { circular dichroism } \\ \mathrm{Chl} & =\text { chlorophyll } \\ \mathrm{FAP} & =\text { filamentous anoxygenic phototroph } \\ \mathrm{LUVOIR} & =\text { Large Ultraviolet/Optical/Infrared Surveyor } \\ \mathrm{NIR} & =\text { near-infrared } \\ \mathrm{PEM} & =\text { photoelastic modulator } \\ \mathrm{UV} & =\text { ultraviolet }\end{aligned}$

